\title{
Host nutrition and infectious disease: an ecological view
}

\author{
Val H Smith ${ }^{1}$, Tyrees P Jones $\mathrm{II}^{2}$, and Marilyn S Smith ${ }^{3}$
}

\begin{abstract}
Nutrition is typically discussed in terms of maintaining a proper diet and avoiding nutrient deficiency diseases. However, nutrition can also be viewed from an ecological standpoint: mammalian hosts and their pathogens share a wide range of resource needs that are required to support normal metabolism and growth. The development of an infectious disease within a host involves the processes of invasion and resource consumption. Competition for growth-limiting resources can potentially occur between the invading pathogen and the cellular components of the host. Experimental studies confirm that nutrients can have profound effects on a host's response to an infection, and taking an ecological view of host nutrition may help to further inform biomedical practice.
\end{abstract}

Front Ecol Environ 2005; 3(5): 268-274

W hen we hear the term "nutrition", we typically envision the components of our food that are necessary for a healthy human diet. From the earliest days of our lives, we are constantly admonished to pay close attention to our eating habits. Increasingly clear evidence suggests that poor nutrition contributes to the development of many debilitating or fatal diseases. For example, nutrientfortified foods and oral nutrient supplements are consumed daily worldwide to help prevent the occurrence of common nutrient deficiency diseases such as anemia (iron deficiency), cretinism and goiter (iodine deficiency), rickets (vitamin D deficiency), and scurvy (vitamin C deficiency). Similarly, veterinarians and the animal production industry pay very close attention to the quality of food that is provided to domestic animals, livestock, and farmed fishes. Many nutrients in the diet are also known to interact either positively or negatively with numerous key medicines (Boullata and Armeni 2004).

Poor diet is known to be a strong risk factor for chronic

In a nutshell:
- Like their hosts, pathogens require nutrient or energy resources
to grow and reproduce
- These resources may at times be reduced to levels that can limit
pathogen growth or damage the host organism
- Host cells and pathogens may share the same resources, and
both may experience resource-limited metabolism and growth
- The host's supply of nutrients may influence a pathogen popu-
lation's growth rate, altering the dynamics of disease via direct
ecological mechanisms

\footnotetext{
${ }^{1}$ Department of Ecology and Evolutionary Biology, University of Kansas, Lawrence, KS 66045 (vsmith@ku.edu); ${ }^{2}$ Department of Biology, Georgia State University, Atlanta, GA 30303; ${ }^{3}$ Department of Microbiology, Molecular Genetics, and Immunology, University of Kansas Medical Center, Kansas City, KS 66160
}

heart disease, stroke, hypertension, diabetes, and many cancers. These five disorders together account for roughly two thirds of all deaths in North America (Wardlaw et al. 1994). In the US, deaths due to infectious diseases declined throughout most of the 20th century; however, substantial year-to-year variation, and recent increases in mortality due to emerging diseases such as HIV, emphasize the dynamic nature of infectious diseases and the need to be prepared to address them (Armstrong et al. 1999). Part of this preparedness should include explorations of the links between nutrition and infectious disease.

If we consider that infected organisms are in fact ecological systems that are being invaded by another organism, the role of host nutrition can be examined from a very unique viewpoint. The host is, of course, a parasite's environment in both the ecological and evolutionary sense (Roberts and Janovy 2005). The development of infectious disease (and its associated signs and symptoms) thus involves the ecological processes of invasion, predator-prey dynamics, resource consumption, and resource competition (cf Bohannan 2000). All infections, no matter how mild, tend to decrease the host organism's nutrient intakes and increase their nutrient losses (Scrimshaw 1991). Although not yet fully understood, the host's nutrient supply can have a strong influence on the growth and dynamics of pathogen populations, and thus on infectious disease-related health. However, current textbooks on the relationships between diet, nutrition, and disease (eg Coulston et al. 2001) typically do not include chapters written from an ecological perspective.

The ability of the host's immune system to respond to a pathogen invasion and the ability of the microbial invader itself to replicate and proliferate both require a constant supply of the nutrients required for metabolism and growth. The available pools of many of these essential biochemical molecules within a host's system may be strongly related to its diet, so the host's consumption of 
these resources probably directly or indirectly influences the population growth of a diverse array of viral, bacterial, fungal, protozoan, and multicellular pathogens (Smith 1993a,b).

The infected host's diet could, for example, affect pathogen mortality rates indirectly, by controlling the availability of vital nutrients required by one or more components of the host's immune system (Figure 1). Both non-specific immune defenses (eg host production of cytokines, complement plasma proteins, or phagocytes), and specific, cellular immune defenses (the production and activity of $B$ and $\mathrm{T}$ lymphocytes), could be influenced by host nutrition. For example, Butcher and

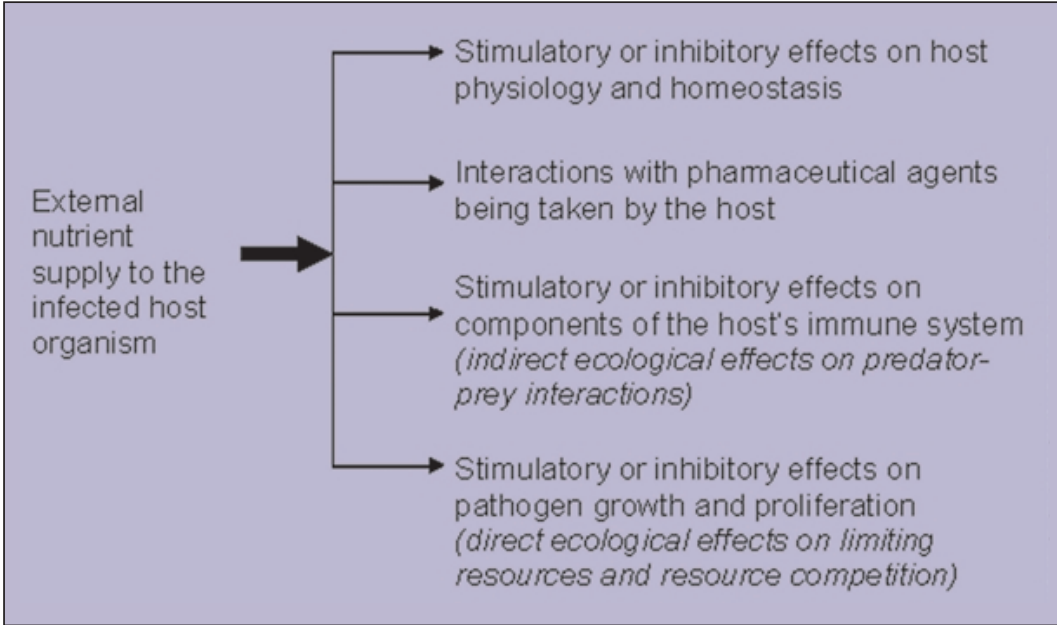

Figure 1. Possible effects of nutrition on an infected mammalian host.
Miles (2002) discuss seven mechanisms by which nutrition might alter the immune response of birds to infective agents, and dietary nutrients have been demonstrated to influence immune function in many vertebrates, including humans (eg Slater and Keymer 1988; Grimble 2001; Jokinen et al. 2003; Kidd 2004). Without doubt, nutritional modulation of host immunity can be a key determinant of host-pathogen interactions; however, this indirect mechanism is not the focus of this paper.

Because many key resource requirements may be identical for host organisms and pathogens, we focus here instead on the direct effects of nutrient supplies on pathogen growth and pathogenesis. Resource availability to invading disease organisms and to cellular components of the mammalian host environment can play a pivotal role in host-pathogen interactions (Weinberg 1978, 1998; Keymer et al. 1983a; Nesheim 1984; Smith 1993a,b; Smith and Holt 1996). In this paper, pathogenesis is viewed as an ecological process in which a pathogen colonizes and persists within the host, producing a population sufficiently numerous, active, and welllocated to exert a localized or systemic pathological effect (Costerton et al. 1987). We provide evidence confirming that the host's diet can profoundly influence the dynamics of pathogen infections, and argue that such nutritional effects can occur via direct ecological mechanisms involving resource supply rates (Figure 1 ). We provide experimental examples of very strong nutrient control of pathogen growth and the outcome of disease, for both microbial and metazoan infections in a variety of mammalian hosts. We also speculate on the future role of nutrition in medicine and ecology.

\section{Protozoan parasites and their effects on host nutrient resource pools}

Pathogenic protozoa are responsible for some of the world's most devastating diseases, and also provide some of the best examples of the strong resource dynamics that accompany pathogen growth within a host organism. The presence of large numbers of active parasites should result in severe drains on the host's internal nutrient pool (Bush et al. 2001). For example, the blood stage of the malaria parasite (Plasmodium spp; see Figure 2a) actively ferments glucose as a primary source of energy, and infected erythrocytes utilize up to 75 times more glucose than uninfected red blood cells (Wiser 1999). As can be seen in Figure 3, an experimental infection of a mouse with Plasmodium chabaudi led to rapid proliferation of the pathogen, and was accompanied by a marked depression in blood glucose levels due to pathogen resource consumption and insulin production by the mouse (Elased and Playfair 1996).

If an invading parasite consumes the bulk of critically important resources such as glucose, the host will suffer (Bush et al. 2001). When the population size of a highly competitive parasite with a high glucose demand expands within the host, the risk of hypoglycemia will increase as host glucose production and intake become insufficient to cover the joint host-parasite glucose demand (Binh et al. 1997). For example, Plasmodium-induced hypoglycemia can be extremely detrimental to the infected host, and a study of 532 cases of severe malaria in India found that mortality was greatest in patients with very low blood glucose levels (less than $2.2 \mathrm{mmol} / \mathrm{l}$ ), and least in patients with blood glucose levels greater than $3.3 \mathrm{mmol} / \mathrm{l}$ (Kochar et al. 1998).

Bush et al. (2001) provide another, even more impressive example of the depression of within-host resources that can accompany rapid protozoan pathogen growth. Mice that have been experimentally infected with virulent African trypanosomes (Trypanosoma brucei) can develop very large parasite populations of about one billion cells $/ \mathrm{ml}$ of blood volume, and death can occur within one week of the injection of a single trypanosome cell. At the time of host death, blood glucose levels approach zero, and liver glycogen is almost totally depleted. The energy resources of the host are insufficient to maintain homeostasis, and the parasite successfully out-competes the host organism for carbohydrates (Bush et al. 2001). In 

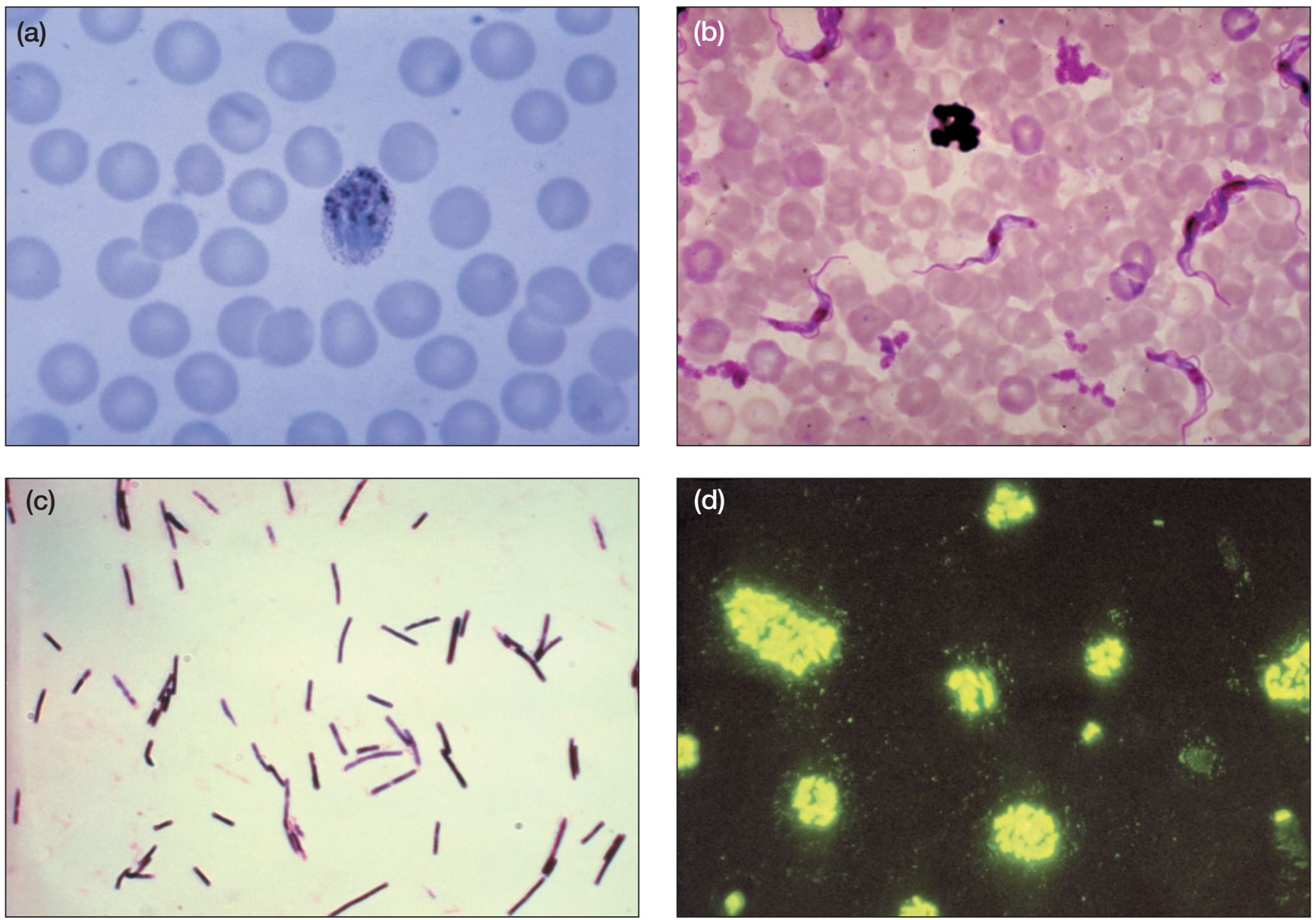

Figure 2. Common mammalian pathogens. (a) Immature schizont of the malaria parasite Plasmodium vivax; (b) the African sleeping sickness parasite Trypanosoma brucei; (c) Clostridium perfringens, the bacterial pathogen responsible for gas gangrene; (d) Salmonella enteriditis, a bacterial pathogen responsible for gastrointestinal infections.

this and other protozoan infections, the exact time course of population growth and glucose depletion should also depend upon the host organism's genetic makeup, and upon the host's pre-infection state in terms of overall health and pre-existing energy stores (Kochar et al. 1998).

\section{Resource effects on pathogen growth and disease severity}

\section{Carbohydrate supply effects}

The above evidence illustrates how the growth of an invading pathogen can in some cases dramatically deplete the host's vital nutrient resource stores, leading to aggravated symptoms and even death. Nutritional support for patients undergoing drug therapy while being treated for severe malaria can include the provision of glucose to help offset the stress of hypoglycemia (Binh et al. 1997), but for some parasitic infections the provision of additional carbohydrates may simply fuel further pathogen growth without altering the ultimate disease outcome. For example, in the trypanosome disease model described by Bush et al. (2001), the infected mouse can be helped to survive for an additional few hours if it is injected with a small volume of glucose solution, but microparasite numbers will double during this same period of time, and host death still ultimately occurs.

Strong stimulation of the growth of a highly competitive pathogen by excessive carbohydrate supplies in the host's diet can also be seen in macroparasites as well. As noted by Bush et al. (2001), both metazoan (multicellular animal) parasites and their hosts are composed of eukaryotic cells, and thus both require very similar resources to grow; the authors also stress that any loss of within-host resources to parasite uptake and metabolism can only be corrected if the host is able to re-supply its nutritional needs.

The extensive literature on parasitic worms in fact provides some of the best experimental evidence for a strong role of host diet in regulating host-pathogen dynamics. For example, Nesheim and coworkers (Nesheim et al. 1978; Nesheim 1984) performed elegant studies of the effect of dietary carbohydrates on the Acanthocephalan parasitic worm Moniliformis dubia in growing rats. Nesheim et al. (1978) varied the starch content of the rats' food, but carefully held the total dietary calorie intake constant. They found that the final parasite content of the gut (mg parasite/mg host 
weight) was linearly dependent upon the proportion of starch provided in the isocaloric food supply, revealing an exceptionally strong dependence of worm growth on the carbohydrate content of the host's diet (Figure 4). Numerous other striking examples can be found in the literature (eg Keymer et al. 1983b; Bush et al. 2001).

\section{Iron supply effects}

Carbohydrates are not the only nutrient resource that can potentially stimulate within-host pathogen growth. For example, iron is an essential metal required for the metabolism and growth of almost all known organisms, in part because of its key role in the cytochrome system. During the past

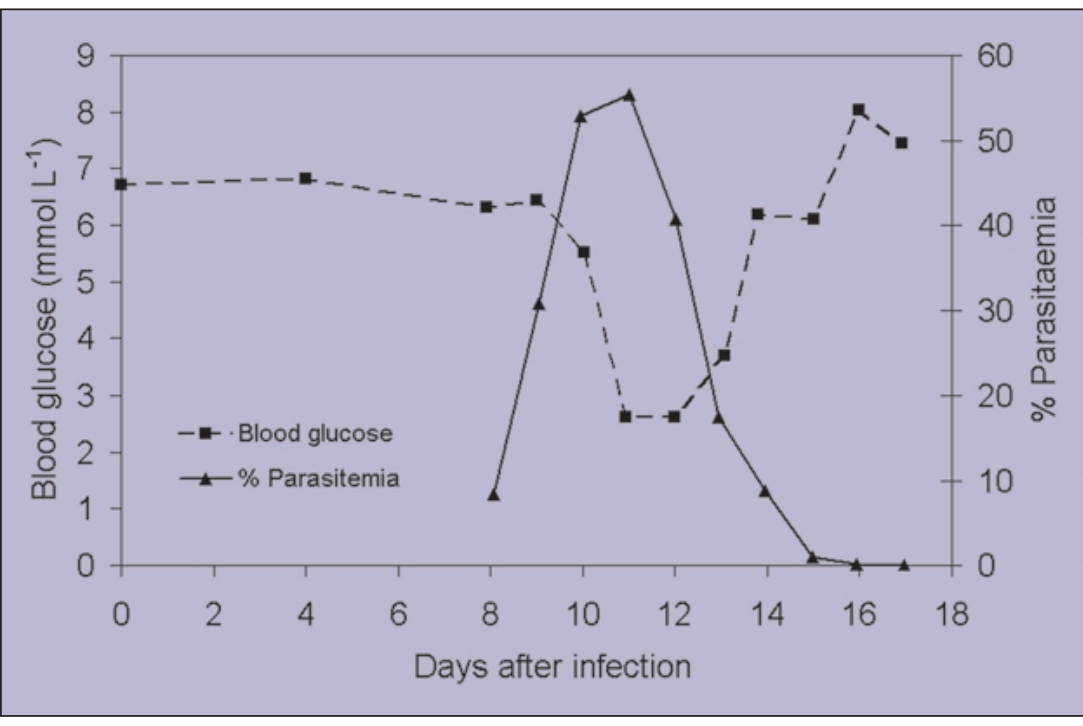

Figure 3. Effects of Plasmodium growth on parasitemia (percent of red blood cells infected) and blood glucose levels ( $\mathrm{mmol} / \mathrm{l})$ in a mouse model of malaria. Data from Elased and Playfair (1996). three decades, many hundreds of publi-

cations have documented the critical role of iron in the establishment and progress of infection in vertebrate hosts (Weinberg and Weinberg 1995). Infectious disease agents whose virulence is enhanced by iron include fungi, protozoa, gram-positive and acid-fast bacteria, and gram-negative bacteria, and this list continues to grow as we learn more about the role of iron in pathogenesis (Weinberg 1999). The exquisite sensitivity of pathogen growth to iron availability is shown in Figure 5. Although the numbers of Clostridium perfringens (Figure 2c) in lab-inoculated mice dropped exponentially in control animals due to rapid control by the host's immune system, the population size of this pathogen was increased by over 50 -fold in the animals that were provided with supplementary iron in their water supply (Bullen et al. 1967).

Even more dramatic evidence of the effects of iron on the outcome of disease is provided by Sword (1966), who worked with a rodent model of Listeria monocytogenes infection. Sword found that variation in the animal's iron supply caused the $\mathrm{LD}_{50}$ (the number of injected bacteria leading to $50 \%$ mortality in the test animals) to be reduced dramatically, from over 37000 cells in control animals to roughly one Listeria monocytogenes cell in animals receiving iron supplementation.

What might be the corresponding implications of high iron intake for human health? The evidence shown in Figure 5 suggests that the consumption of high iron diets, or the absorption of high concentrations of environmental iron could markedly stimulate the growth of invading pathogens. Hundreds of research reports have confirmed that both pathogen growth and the severity of many infectious diseases can be greatly enhanced by high iron availability, whether the excess iron is supplied in the diet, or whether it is absorbed via other mechanisms such as the inhalation of cigarette smoke or through occupational exposures such as those experienced by workers in iron foundries (Weinberg 1998).

\section{Glutamine effects on HIV}

A strong nutrient-dependence of growth can also be seen in viral pathogens. Virus particles lack most of the structural components and biochemical pathways that are shared by bacteria, fungi, protozoa, and multicellular pathogens. However, they are exceptionally parasitic, and following infection they make use of the normal anabolic and catabolic processes of their host cells in order to accomplish viral replication. In doing so, the virus places strong energetic and nutritional demands on its host cells, which in turn will create a drain on the host's nutrient pools. Human immunodeficiency virus type 1 (HIV-1), for example, can be grown in tissue cul-

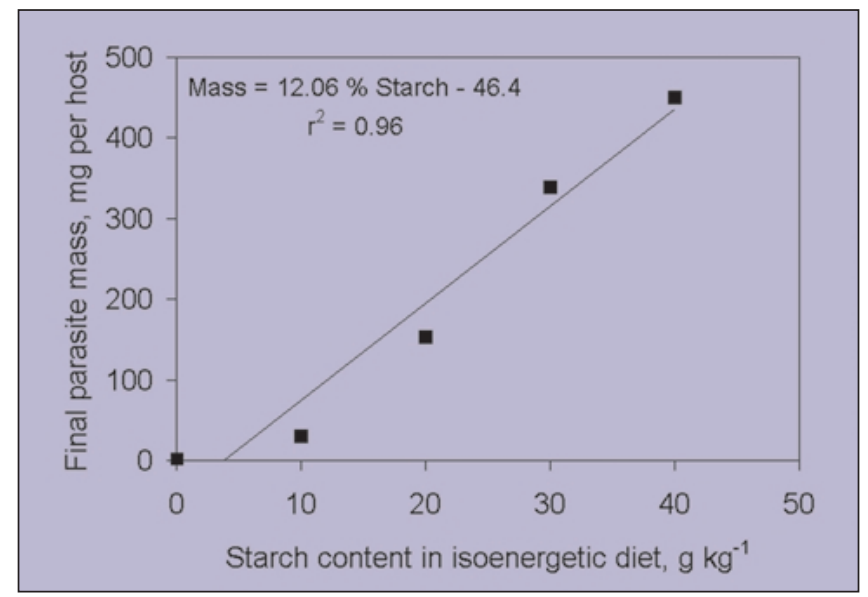

Figure 4. Effects of dietary starch content on final Moniliformis biomass ( $\mathrm{mg} / \mathrm{host})$ in a rat model of disease. Data from Nesheim et al. (1978). 


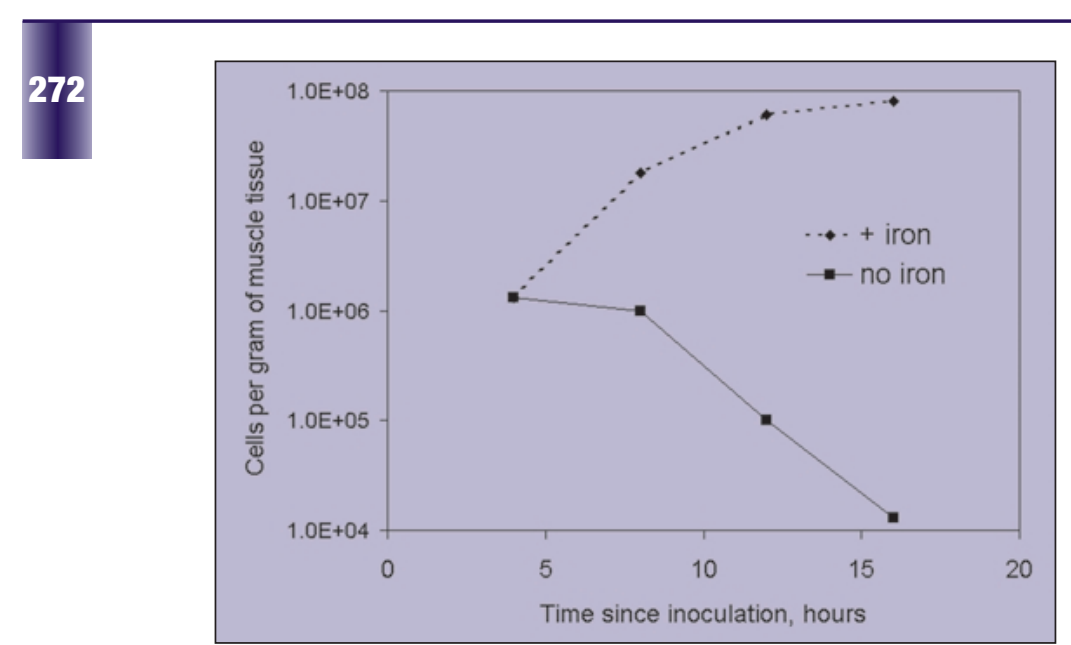

Figure 5. Stimulatory effects of dietary iron on the population dynamics of the pathogenic bacterium Clostidium perfringens in a mouse model of disease. Data from Bullen et al. (1967).

ture only if the cells are provided with periodic exposure to fresh solutions of growth medium. Smith (1993b) proposed that HIV replication can potentially be limited by the supply of the conditionally limiting amino acid glutamine, and experiments using reverse transcription assays indicate that HIV production in human CEM cells (an immortalized T-cell line used in tissue culture) can be profoundly influenced by modifying the concentration of this amino acid in the growth medium (Figure 6). These data provide evidence to support the hypothesis of Smith (1993b) that within-host dynamics of one of the world's most important viral diseases may be sensitive to components of the host's food supply.

\section{Resource effects: pathogen growth restriction and disease control}

Given the apparent sensitivity of very diverse microbial invaders to nutrient availability, it should logically follow that the restriction of critically important nutrients in the

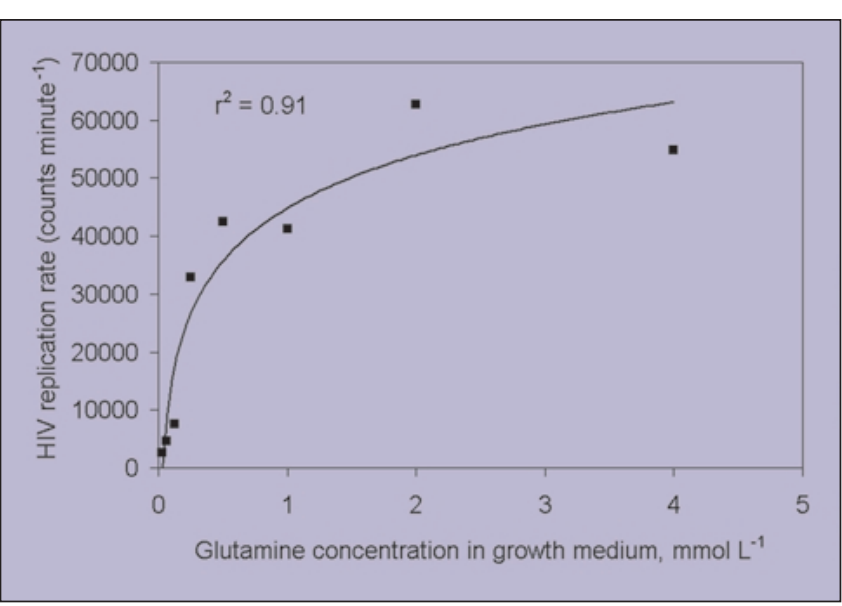

Figure 6. Effects of variations in glutamine supply on the replication of HIV-1 in laboratory-cultured CEM cells. HIV replication was measured using reverse transcriptase assays, and glutamine was provided as the reagent GlutaMax. host's external supply, or reducing the size of their internal nutrient pools, should lead to sharply reduced pathogen growth and alter the outcome of infection. Both responses have in fact been widely and consistently observed for iron. For example, Sword (1966) included two additional treatments in his study of the effects of iron on experimental Listeria infections. He supplemented the water supply of a third set of animals with both iron and desferal, an iron chelating agent; and he supplemented the water supply of a fourth set of animals with the iron chelator alone. Desferal increased the dose of Listeria cells needed to kill $50 \%$ of the population $\left(\mathrm{LD}_{50}\right)$ of iron-treated rats by two orders of magnitude, from less than one cell to 130 cells; moreover, desferal also increased the $\mathrm{LD}_{50}$ of rats receiving no iron to 1.1 million cells, a 30-fold increase relative to control levels. Restriction of dietary iron with desferal thus had very strong protective value against this bacterial pathogen, and this chelator has been used successfully to treat microbial human diseases in a variety of clinical settings (Weinberg 1998, 1999).

The stimulatory effect of high carbohydrate diets seen in Figures 3 and 4 also suggests that the restriction of carbohydrate calories in the diet may help to improve the outcome of many diseases. Evidence to support this hypothesis comes from a study by Peck et al. (1992), who examined the effect of varying protein:carbohydrate ratios in the diet on laboratory infections with the gastrointestinal pathogen Salmonella (Figure 2d). As can be seen in Figure 7, increasing the protein content of the animals' diet resulted in a 50\% reduction in mortality. Dietary energy restriction also influences the outcome of macroparasite infections. For example, Read and Rothman (1957a) found that if rats harboring previously established populations of the cestode parasite Hymenolepis diminuta were placed on a carbohydrate-deficient diet for only 15 days, roughly one-third of the worms were lost from the infected hosts. Strong negative effects of dietary carbohydrate deficiency on parasite burden and egg production were confirmed in subsequent experiments (Read and Rothman 1957b).

\section{Conclusions}

Humans and other mammals are susceptible to a very wide range of infectious diseases, and both human and veterinary medicine rely heavily upon the use of a finite arsenal of antibiotics and antivirals in order to control many of these diseases. However, the incidence of pathogen drug-resistance is increasing worldwide, and many diseases remain for which drug treatments are expensive, limited in availability, or even non-existent. New approaches to the control of infectious disease are badly needed.

The data shown in Figures 3-7 demonstrate unambiguously that the nutrient supply to an infected host can have important, and sometimes profound, effects upon 
the disease process. Weinberg $(1998,1999)$ has for over two decades pointed out the role of iron in disease and the value of iron restriction in disease management, and we believe that his ecological viewpoint can be expanded to other key resources as well. However, in many cases where dietary nutrients have been found to influence either host susceptibility to infection or the actual outcome of disease, the supporting evidence has often been correlative or even anecdotal in nature. The key nutrients that may be involved are typically not identified, and the quantitative nutrient requirements of both the host organism and the pathogen itself are also typically unknown. As the essential resource requirements of different hosts and disease organisms become better quantified, we believe that it should become possible to make predictions concerning the nutrient supply ratios and the absolute nutrient supply rates to the host that should result in the most favorable post-infection outcome (Smith and Holt 1996; Smith and Pippin 1998).

For example, the data shown in Figure 4 suggest that feeding a high-carbohydrate diet to Moniliformis-infected mammalian hosts could be very detrimental, even if they are simultaneously being treated with drugs that are intended to control parasite growth. We suggest here that the actual outcome of infection should depend upon the relative magnitudes of pathogen growth stimulation by dietary carbohydrates, and pathogen mortality imposed by the anti-parasitic drugs. It is conceivable to us that an inappropriately designed diet may result in rapid worm growth that could overwhelm the infected host's immune system, resulting in a catastrophic disease progression or even death. Conversely, it is possible that the provision of an appropriate dietary formulation, designed to restrict resource flow to the invading pathogen during anti-parasite drug therapy, could have a positive and synergistic effect on the animal's health.

Similarly, the effects of providing pure intravenous glucose as nutritional support for critically ill human patients could depend upon the resource requirements of the disease agent that infected them. Figure 7 suggests that for pathogens like Salmonella, the provision of a carefully formulated intravenous nutrient mix with a low carbohydrate:protein content might be a safer and more effective means of providing nutritional support.

In this brief synthetic overview, we have relied primarily upon analyses of previously published data to perform initial tests of the hypothesis that nutrition can alter host-pathogen dynamics via direct ecological mechanisms. However, rigorous and more conclusive tests of this hypothesis will require well replicated in vivo studies that are designed to quantify both the indirect and direct effects of host nutrition on pathogen growth, the severity of disease symptoms, and the final outcome of infection. If dietary nutrients are confirmed to influence the disease process through ecological mechanisms, then we suggest that an ecological view of host nutrition can potentially help to inform biomedical practice and nutrition science.

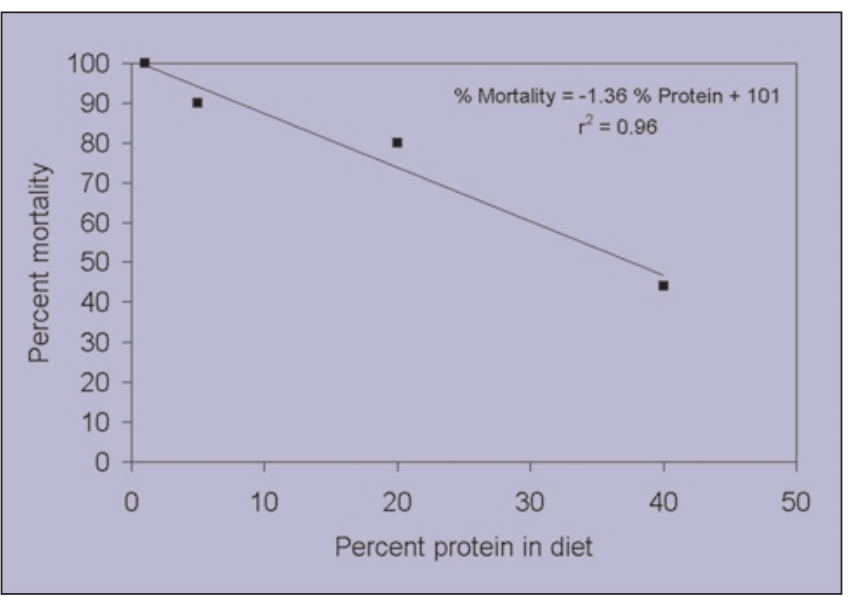

Figure 7. Effects of dietary protein content on percent mortality from Salmonella infection in a rodent model of disease. Data from Peck et al. (1992).

In addition, this research may also help elucidate the degree to which disease and resources may interact as regulators of ecosystem structure and process (eg Cleaveland et al. 2002; Murry et al. 1998; Thomas et al. 2005).

\section{Acknowledgments}

This work was supported in part by NSF grants DMS0342239 to VHS and DMS-0342325 to MSS, and support was provided to TPJ through NSF REU grant DBI0353911. The HIV experiments were performed by MSS while supported as a visiting scientist at the Université de Montréal, Canada.

\section{References}

Armstrong GL, Conn LA, and Pinner RW. 1999. Trends in infectious disease mortality in the United States during the 20th century. J Amer Med Assoc 281: 61-66.

Binh TQ, Davis TM, Johnston W, et al. 1997. Glucose metabolism in severe malaria: minimal model analysis of the intravenous glucose tolerance test incorporating a stable glucose label. Metabolism 46: 1435-40.

Bohannan BJM. 2000. Effect of resource supply rate on host-pathogen dynamics. In: Bell, CR, Brylinsky, M, JohnsonGreen, P (Eds), Microbial biosystems: new frontiers. Proceedings of the 8th International Symposium on Microbial Ecology. Halifax, Canada: Atlantic Canada Society for Microbial Ecology.

Boullata JI and Armeni VT (Eds). 2004. Handbook of drug-nutrient interactions. Totowa, NJ: Humana Press.

Bullen JJ, Cushnie GH, and Rogers HJ. 1967. The abolition of the protective effect of Clostridium welchii type A antiserum by ferric iron. Immunology 12: 303-12.

Bush AO, Fernandez JC, Esch GW, and Seed JR. 2001. Parasitism: the diversity and ecology of animal parasites. Cambridge, UK: Cambridge University Press.

Butcher GD and Miles, RD. 2002. Interrelationship of nutrition and immunity. Gainesville, FL: Veterinary Medicine - Large Animal Clinical Sciences Department, Florida Cooperative Extension Service, Institute of Food and Agricultural Sciences, University of Florida. Document VM139.

Cleaveland S, Hess GR, Dobson AP, et al. 2002. The role of pathogens in biological conservation. In: Hudson PJ, Rizzoli A, 
Grenfell BT, et al. (Eds). The ecology of wildlife diseases. Oxford, UK: Oxford Biology.

Costerton JW, Cheng KJ, Geesey GG, et al. 1987. Bacterial biofilms in nature and disease. Annu Rev Microbiol 41: 435-64.

Coulston AM, Rock CL, and Monsen ER. 2001. Nutrition in the prevention and treatment of disease. New York, NY: Academic Press.

Elased KM and Playfair JHL. 1996. Reversal of hypoglycaemia in murine malaria by drugs that inhibit insulin secretion. Parasitology 112: 515-21.

Grimble RF. 2001. Nutritional modulation of immune function. $P$ Nutr Soc 60: 389-937.

Jokinen EI, Vielma J, Aaltonen TM, and Koskela J. 2003. The effect of dietary phosphorus deficiency on the immune responses of European whitefish (Coregonus lavaretus L). Fish Shellfish Immun 15: 159-68.

Keymer A, Crompton DWT, and Walters DE. 1983a. Nippostrongylus (Nematoda) in protein-malnourished rats: host mortality, morbidity and rehabilitation. Parasitology 86: 461-75.

Keymer A, Crompton DWT, and Walters DE. 1983b. Parasite population biology and host nutrition: dietary fructose and Moniliformis (Acanthocephala). Parasitology 87: 265-78.

Kidd MT. 2004. Nutritional modulation of immune function in broilers. Poultry Sci 83: 650-57.

Kochar DK, Thanvi I, Kumawat BL, and Shubhakaran AN. 1998. Importance of blood glucose level at the time of admission in severe and complicated malaria. J Assoc Physician India 46: 921-22.

Murray DL, Keith LB, and Cary JR. 1998. Do parasitism and nutritional status interact to affect production in snowshoe hares? Ecology 79: 1209-22.

Nesheim MC, Crompton DW, Arnold S, and Barnard D. 1978. Host dietary starch and Moniliformis (Acanthocephala) in growing rats. P Phys Soc Lond B 202: 399-408.

Nesheim MC. 1984. Some experimental approaches to the study of nutrition and parasitic infection. Fed Proc 43: 235-38.

Peck MD, Babcock GF, and Alexander JW. 1992. The role of protein and calorie restriction in outcome from Salmonella infec- tion in mice. J Parenter Enter Nutr 16: 561-65.

Read CP and Rothman AH. 1957a. The role of carbohydrates in the biology of cestodes. I. The effect of dietary carbohydrate quality on the size of Hymenolepis diminuta. Exp Parasitol 6: 1-7.

Read CP and Rothman AH. 1957b. The role of carbohydrates in the biology of cestodes. IV. Some effects of host dietary carbohydrate on growth and reproduction of Hymenolepis. Exp Parasitol 6: 294-305.

Roberts LS and Janovy J Jr. 2005. Foundations of parasitology, 7th edn. New York, NY: McGraw-Hill.

Scrimshaw NS. 1991. Effect of infection on nutrient requirements. J Parenter Enter Nutr 15: 589-600.

Slater AFG and Keymer AE. 1988. The influence of protein deficiency on immunity to Heligmosomoides polygyrus (Nematoda) in mice. Parasite Immunol 10: 507-22.

Smith VH. 1993a. Resource competition between host and pathogen. BioScience 43: 21-31.

Smith VH. 1993b. Implications of resource-ratio theory for microbial ecology. Adv Microb Ecol 13: 1-37.

Smith VH and Holt RD. 1996. Resource competition and withinhost disease dynamics. Trends Ecol Evol 11: 386-89.

Smith VH and Pippin DJ. 1998. Implications of resource-ratio theory for oral microbial ecology. Eur J Oral Sci 106: 605-15.

Sword CP. 1966. Mechanisms of pathogenesis in Listeria monocytogenes infection. J Bacteriol 92: 536-42.

Thomas F, Renaud F, and Guégan J-F (Eds). 2005. Parasitism and ecosystems. New York, NY: Oxford University Press.

Wardlaw GM, Insel PM, and Seyler MF. 1994. Contemporary nutrition: issues and insights, 2nd edn. St. Louis, MO: Mosby.

Weinberg ED. 1978. Iron and infection. Microbiol Rev 42: 45-66.

Weinberg ED. 1998. Patho-ecologic implications of microbial acquisition of host iron. Rev Med Microbiol 9: 171-78.

Weinberg ED. 1999. The role of iron in protozoa and fungal infectious diseases. J Eukaryot Microbiol 46: 231-38.

Weinberg ED and Weinberg GA. 1995. The role of iron in infection. Curr Opin Infect Dis 8: 164-69.

Wiser MF. 1999. Biochemistry of Plasmodium - brief overview. www.tulane.edu/ wiser/malaria/Summary.html\#sub. Viewed 2 August 2004. 